\title{
The detrimental effect of spontaneous emission in quantum free electron lasers: A discrete Wigner model
}

H. Fares, N. Piovella, and G. R. M. Robb

Citation: Physics of Plasmas 25, 013111 (2018); doi: 10.1063/1.5003913

View online: https://doi.org/10.1063/1.5003913

View Table of Contents: http://aip.scitation.org/toc/php/25/1

Published by the American Institute of Physics

\section{Articles you may be interested in}

Theory of relativistic radiation reflection from plasmas

Physics of Plasmas 25, 013108 (2018); 10.1063/1.5000785

Prospects and limitations of wakefield acceleration in solids

Physics of Plasmas 25, 013107 (2018); 10.1063/1.5003857

Radiation pressure injection in laser-wakefield acceleration

Physics of Plasmas 25, 013110 (2018); 10.1063/1.5006325

Self-focusing and defocusing of Gaussian laser beams in collisional inhomogeneous plasmas with linear density and temperature ramps

Physics of Plasmas 25, 012309 (2018); 10.1063/1.5007800

The role of the global phase in the spatio-temporal evolution of strong-coupling Brillouin scattering

Physics of Plasmas 25, 013114 (2018); 10.1063/1.5019374

Relativistic extension of a charge-conservative finite element solver for time-dependent Maxwell-Vlasov equations

Physics of Plasmas 25, 013109 (2018); 10.1063/1.5004557

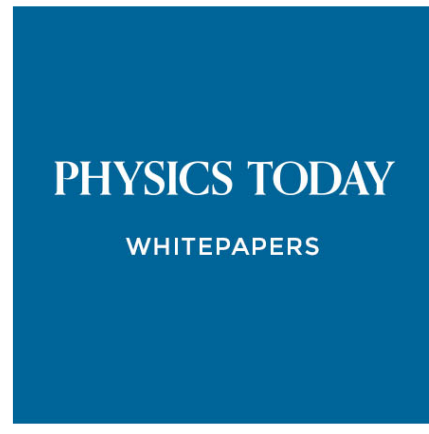

ADVANCES IN PRECISION MOTION CONTROL

Piezo Flexure Mechanisms and Air Bearings
READ NOW

PRESENTED BY

PI 


\title{
The detrimental effect of spontaneous emission in quantum free electron lasers: A discrete Wigner model
}

\author{
H. Fares, ${ }^{1}$ N. Piovella, ${ }^{2}$ and G. R. M. Robb ${ }^{3}$ \\ ${ }^{1}$ INFN-LNF, Via Enrico Fermi, 40, Frascati, 00044 Roma, Italy and Department of Physics, Faculty of \\ Science, Assiut University, Assiut 71516, Egypt \\ ${ }^{2}$ Dipartimento di Fisica, Università degli Studi di Milano, via Celoria 16, I-20133 Milano, Italy \\ ${ }^{3}$ Department of Physics, SUPA, University of Strathclyde, Glasgow, G4 ONG Scotland, United Kingdom
}

(Received 8 September 2017; accepted 21 December 2017; published online 16 January 2018)

\begin{abstract}
We study the spontaneous emission in high-gain free-electron lasers operating in the quantum regime and its detrimental effect on coherent emission. A quantum model describing the coherent and spontaneous emission in free electron lasers has been recently proposed and investigated [G. R. M. Robb and R. Bonifacio, Phys. Plasmas 19, 073101 (2012)]. The model is based on a Wigner distribution describing the electron beam dynamics, coupled to Maxwell equations for the emitted radiation field. Here, we rephrase the model in a more rigorous way, considering a discrete Wigner distribution defined for a periodic space coordinate for which the electron momentum is discrete. From its numerical solution, we find good agreement with the approximate continuous model. In the quantum regime of the free-electron laser, we obtain a simple density matrix equation for two momentum states, where the role of the spontaneous emission has a clear interpretation in terms of coherence decay and population transfer. Published by AIP Publishing. https://doi.org/10.1063/1.5003913
\end{abstract}

\section{INTRODUCTION}

Free electron lasers (FELs) using highly relativistic electron beams passing through very long magnetic undulators are currently operating as high-intensity coherent x-ray radiation sources, with many interesting applications. ${ }^{1,2}$ A proposed extension of these machines includes the use of laser wigglers ${ }^{3}$ or micro-undulators, ${ }^{4}$ in order to make such devices more compact and flexible. In these new schemes, the quantum recoil associated with emission of each photon starts to play an important role, since the photon recoil can be comparable with the fraction of the electron momentum transferred to the radiation. From this perspective, the Quantum FEL (QFEL) concept ${ }^{5,6}$ is attractive as a potential source of intense, quasi-monochromatic radiation at wavelengths in the Angstrom or even sub-Angstrom range.

The identification of a quantum or classical regime of FEL operation is characterized by a dimensionless parameter $\bar{\rho}$ introduced by Bonifacio et al., ${ }^{5}$ equal to the ratio of the induced momentum spread $\delta p_{z} \sim m c \gamma \rho$ (where $\rho$ is the FEL parameter $^{7}$ ) to the photon momentum $\hbar k$. An FEL operates in the quantum regime when $\bar{\rho}<1$, such that each electron emits a single photon in a transition between two momentum states. Correspondingly, the spectrum of the QFEL is expected to reduce to a single, narrow line. When $\bar{\rho} \gg 1$, the quantum discreteness of the changes in electron momentum due to photon emission has no effect on the FEL operation. Consequently, multiple transitions of electrons between momentum states produce the broad and spiky spectrum expected from the classical FEL theory. In the latter case, the classical description of the FEL involving a collection of particle-like electrons is adequate to describe the FEL dynamics.

In FEL-based light sources operating at short or ultrashort wavelengths, the spontaneous emission sets an intrinsic limit on the coherent production of photons, due to the growth of the induced energy spread in the electron beam. Pioneering classical studies on spontaneous emission by highly relativistic electron beams in magnetic undulators, and its induced energy spread, have been reported in Refs. 8 and 9, but only recently has a quantum model of such spontaneous emission processes been proposed in Ref. 10, where the evolution of the electron momentum distribution occurs as discrete momentum groups described by a Poisson distribution. In subsequent works, ${ }^{11,12}$ a self-consistent quantum FEL model including spontaneous emission was presented and the criteria for neglecting its detrimental effect on the coherent FEL operation were derived.

In Refs. 11 and 12, an equation based on a continuous Wigner distribution was used to describe the electron dynamics, as will be reviewed shortly in Sec. II. Here, we further investigate the role of the spontaneous emission in FELs using a model based on a discrete Wigner function. ${ }^{16,17}$ We study in detail the electron dynamics and the radiation growth along the undulator. Furthermore, we present the results of a linear analysis, from which we can estimate the effect of the spontaneous emission on the growth rate of the intensity.

For highly relativistic beams passing through an undulator, each spontaneous photon is in general emitted by an electron with energy $m c^{2} \gamma$ at a random angle $\phi$ with respect to the electron beam direction $z{ }^{18}$ As a consequence, the frequency distribution of the spontaneous radiation is not purely monochromatic, but has a broadband distribution $g(\nu)=(3 / 2)$ $\left(1-2 \nu+2 \nu^{2}\right)$, where $\nu=k(\phi) / k(\phi=0)=1 /\left(1+\gamma^{2} \phi^{2}\right)$. The spontaneous radiation emitted by a relativistic electron has been studied in several papers investigating inverse Compton sources, ${ }^{13,14}$ and the impact of the electron recoil on the radiation bandwidth has been discussed in Ref. 15. A proper description of the role of the spontaneous emission in quantum FELs should take into account its frequency distribution, leading in general to an electron momentum distribution, which is not 
perfectly discrete, but consists of lines with some broadening. Clearly, a realistic description of a QFEL experiment would also require a description of full $3 \mathrm{D}$ effects, including diffraction of the laser wiggler, off-axis emission, and finite emittance of the electron beam. However, in order to focus interest on the effect of the spontaneous emission in the QFEL operation only, the 1D approximation, also if rather crude, is useful to understand its basic physical mechanism. Furthermore, we point out that it has been already shown in Ref. 12 that the inclusion of broadband spontaneous emission does not significantly affect the competition between incoherent spontaneous emission and coherent spontaneous emission when the FEL operates in the quantum regime. On the basis of these results, we will describe the spontaneous emission as effectively monochromatic radiation, neglecting its broadband spectral nature for simplicity. This implies the assumption that the electron momentum distribution consists of discrete states separated by multiples of $\hbar k$.

This paper is organized as follows: In Sec. II, for consistency, we review the continuous Wigner model as described in Ref. 11. In Sec. III, we present an analysis of FELs in terms of a discrete Wigner function, ${ }^{16}$ with the inclusion of spontaneous emission. Using this model, we describe the coherent and incoherent emission in an FEL, where the radiation is assumed to be monochromatic and emitted along the undulator axis $z$. In Sec. IV, the equations describing the QFEL in the quantum regime are derived. Finally, the numerical results are presented in Sec. V.

\section{CONTINUOUS WIGNER MODEL}

As described in Ref. 10, the spontaneous emission involves the emission of photons with momentum $\hbar k$ (where $k=2 \pi / \lambda$ is the photon wavenumber directed along the $z$-axis) at a rate $R=\alpha k_{w} a_{w}^{2} / 3$ per unit distance through the undulator, where $a_{w}=e B_{w} / k_{w} m c\left(a_{w} \ll 1\right)$ is the undulator parameter, $k_{w}=2 \pi / \lambda_{w}, \lambda_{w}$ is the undulator period and $\alpha$ is the fine structure constant. Consequently, the probability of an electron having momentum $p_{z}$ will be increased by spontaneous emission from electrons with momentum $p_{z}+\hbar k$ but decreased by spontaneous emission from electrons with momentum $p_{z}$.

Since the spontaneous emission is described by a rate equation for the electron momentum probability, the previous quantum FEL model based on a Schrodinger-like equation describing the electron beam has been extended to a Wigner distribution $W\left(z, p_{z}\right)$ able to describe two processes: (a) the coherent back-scattering of the undulator pseudophotons (inducing a spatial modulation on the scale of the radiation wavelength, i.e., bunching) and (b) the incoherent change of momentum by units of photon recoil $\hbar k$ due to spontaneous emission, and described by a discrete momentum rate equation. Following Ref. 11, the equations that describe the evolution of the system are as follows:

$$
\begin{aligned}
\frac{\partial W(\theta, \bar{p})}{\partial \bar{z}}+\bar{p} \frac{\partial W(\theta, \bar{p})}{\partial \theta}= & \bar{\rho}\left(A e^{i \theta}+\text { c.c. }\right) \\
& \times\left[W\left(\theta, \bar{p}+\frac{1}{2 \bar{\rho}}\right)-W\left(\theta, p-\frac{1}{2 \bar{\rho}}\right)\right] \\
& +\frac{\beta}{\bar{\rho}}\left[W\left(\theta, \bar{p}+\frac{1}{\bar{\rho}}\right)-W(\theta, \bar{p})\right]
\end{aligned}
$$

$$
\frac{d A}{d \bar{z}}=\int_{-\infty}^{+\infty} d \theta \int_{-\infty}^{+\infty} W(\theta, \bar{p}) e^{-i \theta} d \bar{p}+i \delta A .
$$

Equations (1) and (2) are written in terms of the same dimensionless variables of Ref. $6: \bar{z}=2 k_{w} \rho z$ is the scaled position in the undulator, $\theta=\left(k+k_{w}\right) z-\omega t$ is the ponderomotive electron phase, $\bar{p}=m c\left(\gamma-\gamma_{0}\right) /(\hbar k \bar{\rho})$ is the relative electron momentum in units of $\hbar k \bar{\rho}, A=\sqrt{\epsilon_{0} / n_{e} \hbar \omega \bar{\rho}} E$ is the dimensionless amplitude of the coherent FEL radiation field (such that $\bar{\rho}|A|^{2}$ is the average number of photons emitted per electron), $n_{e}=I / e c 2 \pi \sigma_{e}^{2}$ is the electron density (where $I$ is the peak current and $\sigma_{e}$ is the rms size of a transversely Gaussian beam), $\rho=\left(1 / 2 \gamma_{r}\right)\left(I / I_{A}\right)^{1 / 3}\left(\lambda_{w} a_{w} / 2 \pi \sigma_{e}\right)^{2 / 3}$ (where $I_{A}=17 \mathrm{kA}$ is the Alfvèn current) and $\bar{\rho}=\rho\left(m c \gamma_{r} / \hbar k\right)$ are the classical and quantum FEL parameters, $\delta=\left(\gamma_{r}-\gamma_{0}\right) / \gamma_{r} \rho$ is the detuning, where $\gamma_{0}$ and $\gamma_{r}=\sqrt{k\left(1+a_{w}^{2}\right) / 2 k_{w}}$ are the initial and resonant electron energies in units of $m c^{2}$ and $\beta$ $=\alpha a_{w}^{2} m c \gamma_{r} /(6 \hbar k)$ is the scaled spontaneous emission rate.

\section{DISCRETE WIGNER MODEL}

In Eqs. (1) and (2), the space coordinate is unbounded, i.e., $\theta \in(-\infty,+\infty)$, although in Refs. 11 and 12 , the equations have been solved numerically restricting the space coordinate $\theta$ to a single ponderomotive period $(0,2 \pi]$. Although, in a classical theory, the choice of the $\theta$-domain has no consequences for the conjugate momentum variable $p=\bar{\rho} \bar{p}$, and in a quantum description, $\theta$ and $p$ are intrinsically related: in fact, if $\theta$ is a periodic variable in $(0,2 \pi]$, then necessarily the conjugate momentum variable $p$ is discrete. A more rigorous approach to an FEL Wigner model with periodic boundary conditions in $\theta$ has been presented in Ref. 17, where a discrete Wigner model has been derived for FELs. Here, we extend this model adding to it the "narrowband" spontaneous emission, in a similarly way as realized for the continuous Wigner model of Eqs. (1) and (2).

The equations for the discrete Wigner model are

$$
\begin{gathered}
\frac{\partial w_{s}(\theta)}{\partial \bar{z}}+\frac{s}{\bar{\rho}} \frac{\partial w_{s}(\theta)}{\partial \theta}= \\
\bar{\rho}\left(A e^{i \theta}+A^{*} e^{-i \theta}\right)\left\{w_{s+1 / 2}(\theta)-w_{s-1 / 2}(\theta)\right\} \\
+\frac{\beta}{\bar{\rho}}\left\{w_{s+1}(\theta)-w_{s}(\theta)\right\} \\
\frac{d A}{d \bar{z}}=\sum_{m=-\infty}^{+\infty} \int_{-\pi}^{+\pi} w_{m+1 / 2}(\theta) e^{-i \theta} d \theta+i \delta A
\end{gathered}
$$

where $s=m$ or $s=m+1 / 2$ and $m \in Z$. Here, the momentum is discrete and two separate Wigner functions, for integer and semi-integer indices, are needed. The marginal distributions for the momentum $p_{z}=m(\hbar k)$ and the position $\theta$ are

$$
\begin{gathered}
P_{m}(\bar{z})=\int_{-\pi}^{\pi} w_{m}(\theta, \bar{z}) d \theta \\
Q(\theta, \bar{z})=\sum_{m=-\infty}^{+\infty}\left[w_{m}(\theta, \bar{z})+w_{m+1 / 2}(\theta, \bar{z})\right] .
\end{gathered}
$$

Since $w_{s}(\theta, \bar{z})$ is periodic in $\theta$, it can be expanded in a Fourier series 


$$
w_{s}(\theta, \bar{z})=\frac{1}{2 \pi} \sum_{k=-\infty}^{+\infty} w_{s}^{k}(\bar{z}) e^{i k \theta} .
$$

In particular, $w_{m}^{0}$ is the population of the $m$-th momentum state and $w_{m+1 / 2}^{1}$ is the $m$-th bunching component. From Eqs. (3) to (7), we obtain the equations

$$
\begin{aligned}
\frac{d w_{s}^{k}}{d \bar{z}}+i k \frac{s}{\bar{\rho}} w_{s}^{k}= & \bar{\rho}\left[A\left(w_{s+1 / 2}^{k-1}-w_{s-1 / 2}^{k-1}\right)\right. \\
& \left.+A^{*}\left(w_{s+1 / 2}^{k+1}-w_{s-1 / 2}^{k+1}\right)\right]+\frac{\beta}{\bar{\rho}}\left\{w_{s+1}^{k}-w_{s}^{k}\right\} \\
& \frac{d A}{d \bar{z}}=\sum_{m=-\infty}^{+\infty} w_{m+1 / 2}^{1}+i \delta A
\end{aligned}
$$

\section{THE QUANTUM FEL REGIME}

In the quantum regime, $\bar{\rho} \ll 1$, the momentum space is spanned only by the two states $m=0$ and $m=-1$. Keeping only the terms with $s=0, s=-1$ and $s=-1 / 2$, Eqs. (8) and (9) reduce to

$$
\begin{gathered}
\frac{d w_{0}^{0}}{d \bar{z}}=-\bar{\rho}\left[A w_{-1 / 2}^{-1}+A^{*} w_{-1 / 2}^{1}\right]-\frac{\beta}{\bar{\rho}} w_{0}^{0}, \\
\frac{d w_{-1}^{0}}{d \bar{z}}=\bar{\rho}\left[A w_{-1 / 2}^{-1}+A^{*} w_{-1 / 2}^{1}\right]+\frac{\beta}{\bar{\rho}}\left\{w_{0}^{0}-w_{-1}^{0}\right\}, \\
\frac{d w_{-1 / 2}^{1}}{d \bar{z}}=\frac{i}{2 \bar{\rho}} w_{-1 / 2}^{1}+\bar{\rho}\left[A\left(w_{0}^{0}-w_{-1}^{0}\right)+A^{*}\left(w_{0}^{2}-w_{-1}^{2}\right)\right] \\
-\frac{\beta}{\bar{\rho}} w_{-1 / 2}^{1}, \\
\frac{d A}{d \bar{z}}=w_{-1 / 2}^{1}+i \delta A .
\end{gathered}
$$

Defining the populations of the two momentum states $P_{0}$ $=w_{0}^{0}$ and $P_{-1}=w_{-1}^{0}$, and the bunching variable $B=w_{-1 / 2}^{1}$ (with $B^{*}=w_{-1 / 2}^{-1}$ ) and neglecting the higher spatial harmonic components $w_{0}^{2}$ and $w_{-1}^{2}$, Eqs. (10)-(13) take a form which resembles the Optical Bloch equations for a two-level system

$$
\begin{gathered}
\frac{d P_{0}}{d \bar{z}}=-\bar{\rho}\left(A B^{*}+A^{*} B\right)-\frac{\beta}{\bar{\rho}} P_{0}, \\
\frac{d P_{-1}}{d \bar{z}}=\bar{\rho}\left(A B^{*}+A^{*} B\right)+\frac{\beta}{\bar{\rho}}\left(P_{0}-P_{-1}\right), \\
\frac{d B}{d \bar{z}}=\frac{i}{2 \bar{\rho}} B+\bar{\rho} A\left(P_{0}-P_{-1}\right)-\frac{\beta}{\bar{\rho}} B, \\
\frac{d A}{d \bar{z}}=B+i \delta A .
\end{gathered}
$$

Equations (14)-(17) describe the dynamics of the quantum FEL regime in the two-level approximation. The spontaneous emission has two main effects on the FEL dynamics: (a) it causes the decay of the bunching $B$ (i.e., of the coherence between the two momentum states) and (b) it causes additional transitions from the momentum states $m=0$ and $m=-1$ to lower momentum states, with a rate $\beta / \bar{\rho}$, as described by the last terms of Eqs. (14) and (15).

Redefining the variables as $A^{\prime}=\sqrt{\bar{\rho}} A e^{-i \delta \bar{z}}, B^{\prime}=B e^{-i \delta \bar{z}}$, and $z^{\prime}=\sqrt{\bar{\rho}} \bar{z}$, the above equations become

$$
\begin{gathered}
\frac{d P_{0}}{d z^{\prime}}=-\left(A^{\prime} B^{* *}+\text { c.c. }\right)-D P_{0}, \\
\frac{d P_{-1}}{d z^{\prime}}=\left(A^{\prime} B^{\prime *}+\text { c.c. }\right)+D\left(P_{0}-P_{-1}\right), \\
\frac{d B^{\prime}}{d z^{\prime}}=-i \delta^{\prime} B^{\prime}+A^{\prime}\left(P_{0}-P_{-1}\right)-D B^{\prime}, \\
\frac{d A^{\prime}}{d z^{\prime}}=B^{\prime},
\end{gathered}
$$

where $\delta^{\prime}=[\delta-1 /(2 \bar{\rho})] / \sqrt{\bar{\rho}}$ and $D=\beta / \bar{\rho}^{3 / 2}$. Notice that with these definitions, $\left|A^{\prime}\right|^{2}$ represents the average number of photons emitted per electron. Without spontaneous emission, i.e., for $D=0$, the equations have the following analytic solution at resonance $\left(\delta^{\prime}=0\right),{ }^{17,19}$ for $P_{0}(0)=1, P_{-1}(0)$ $=0, A^{\prime}(0)=0$, and $B^{\prime}(0) \ll 1$ :

$$
\begin{gathered}
A^{\prime}\left(z^{\prime}\right)=\operatorname{sech}\left(z^{\prime}-z_{0}\right), \\
B\left(z^{\prime}\right)=-\sinh \left(z^{\prime}-z_{0}^{\prime}\right) \operatorname{sech}^{2}\left(z^{\prime}-z_{0}^{\prime}\right),
\end{gathered}
$$

with $z_{0}^{\prime} \approx-\ln [B(0) / 4]$. Hence, the maximum emission is reached at $z^{\prime}=z_{0}^{\prime}$ where each electron emits a single photon, such that $\left|A^{\prime}\left(z_{0}^{\prime}\right)\right|^{2}=1$, and the maximum bunching is $1 / 2$ at $z^{\prime}=z_{0}^{\prime} \pm 0.88$, corresponding to the maximum overlap between the two momentum states, with $P_{0}=P_{-1}$. Equations (18)-(21) have the same form of the Maxwell-Bloch equations, well known in quantum optics, ${ }^{20}$ where the electron dynamics is described by a density operator obeying a master equation with a coherent part, ruled by the FEL interaction, and a dissipative part, due in this case to spontaneous emission.

\section{NUMERICAL RESULTS}

In this section, we investigate numerically the effect of spontaneous emission on the FEL operation. Considering the quantum regime, we solved Eqs. (18)-(21) assuming resonance $\left(\delta^{\prime}=0\right)$ and the initial conditions $B^{\prime}(0)=0.01$, $A^{\prime}(0)=0, P_{0}(0)=1$ and $P_{-1}(0)=0$ such that for $D=0$ we have $z_{0}^{\prime} \approx 6$. In Fig. 1 , we plot the average number of photons emitted per electron, $\left|A^{\prime}\right|^{2}$, vs. $z^{\prime}$ for different values of $D$. It is evident from Fig. 1 that the detrimental effects of the spontaneous emission on the coherent FEL emission is negligible if

$$
D=\frac{\beta}{\bar{\rho}^{3 / 2}} \ll 1 .
$$

For $D=0$, the system shows a periodic behaviour, with the photon number emitted per electron reaching the maximum $\left|A^{\prime}\right|^{2}=1$ at $z^{\prime} \sim 6$. Increasing $D$, the quantum efficiency decreases, and it is almost zero for $D>0.2$. 


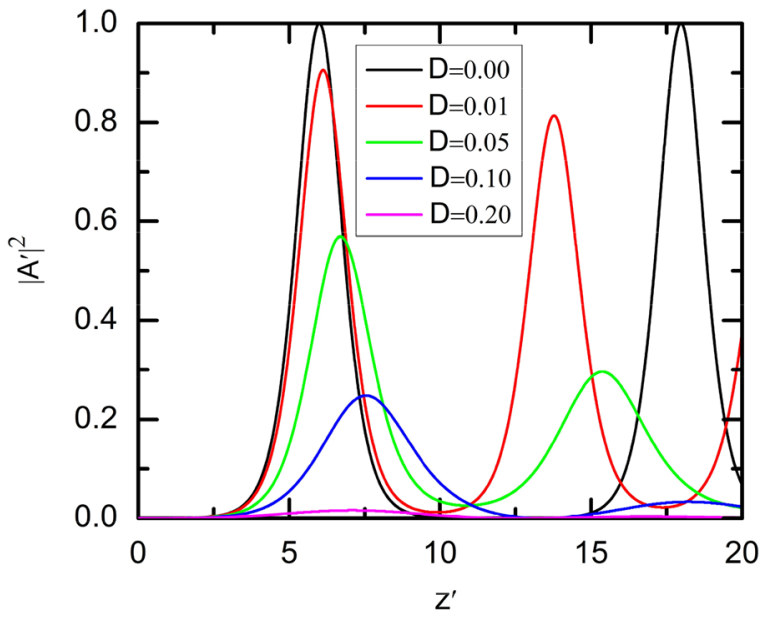

FIG. 1. Scaled intensity $\left|A^{\prime}\right|^{2}$ vs. $z^{\prime}$ in the quantum regime, for different values of the spontaneous emission rate $D$.

We can also study the growth rate of the radiation intensity linearizing Eqs. (14)-(21) around the initial condition with $A^{\prime}(0)=0, B^{\prime}(0)=0, P_{0}(0)=1$ and $P_{-1}(0)=0$. The "zero-order" solution (for $A^{\prime}=0$ ) is $P_{0}\left(z^{\prime}\right)=e^{-D z^{\prime}}$ and $P_{-1}\left(z^{\prime}\right)=D z^{\prime} e^{-D z^{\prime}}$. Then, the linear regime is described by the following equation:

$$
\frac{d^{2} A^{\prime}}{d z^{\prime 2}}+\left(i \delta^{\prime}+D\right) \frac{\tilde{d} A^{\prime}}{d z^{\prime}}-\left(1-D z^{\prime}\right) e^{-D z^{\prime}} A^{\prime}=0 .
$$

For $D=0$ and $\delta^{\prime}=0$, the intensity $\left|A^{\prime}\right|^{2}$ grows exponentially as $\exp \left(g z^{\prime}\right)$, whereas when $D \neq 0$ the equation is nonhomogeneous, and the intensity decrease is not exponential. This can be seen easily in Fig. 2, which shows a plot of $g$ $=\frac{2}{\left|A^{\prime}\right|}\left|\frac{d A^{\prime}}{d z^{\prime}}\right|$ vs. $D$ at resonance (i.e., $\delta^{\prime}=0$ ) for different values of $z^{\prime}$ (notice that in this case $A^{\prime}$ is real). Whereas in a pure exponential regime $g$ should be independent on $z^{\prime}$, here it decreases with $z^{\prime}$.

A comparison between the linear and nonlinear solution in the quantum regime is presented in Fig. 3, where $\left|A^{\prime}\right|^{2}$ is drawn as a function of $z^{\prime}$ for $D=0,0.05,0.1$ for the nonlinear (full lines) and linear (dashed lines) solution. In Fig. 4, we plot the first maximum of $\left|A^{\prime}\right|^{2}$ as a function of $D$. The

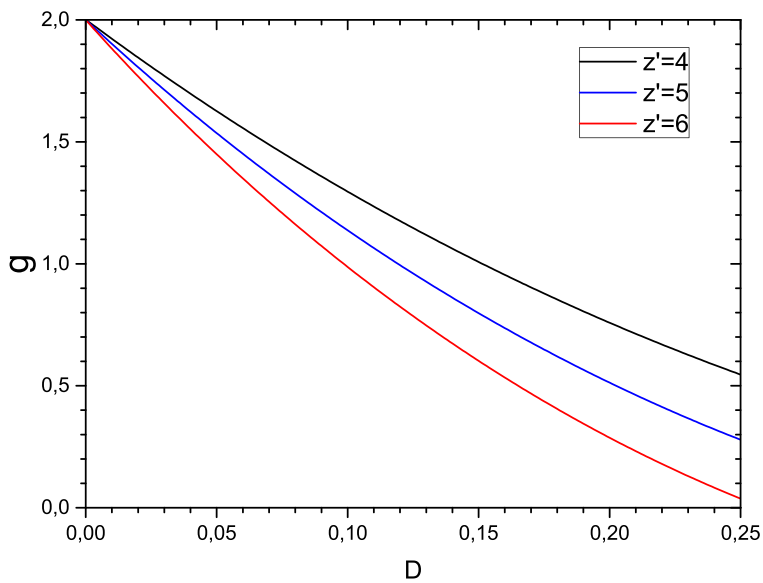

FIG. 2. Growth rate $g=\frac{2}{\left|A^{\prime}\right|}\left|\frac{d A^{\prime}}{d z^{\prime}}\right|$ vs. $D$ in the quantum regime, for three different positions $z^{\prime}$.

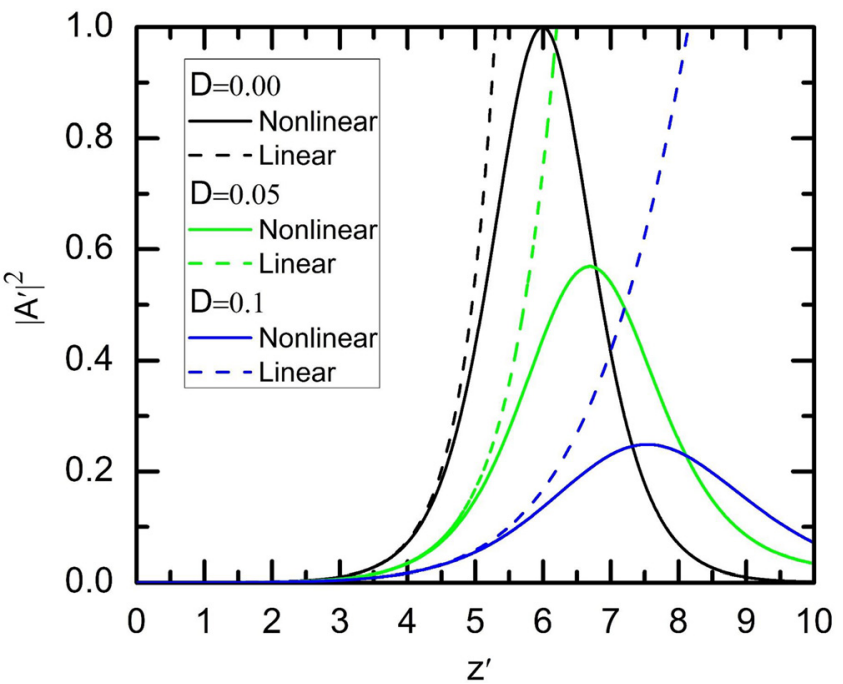

FIG. 3. Comparison between the nonlinear solution (full lines) and the linear solution (dashed lines) for $D=0,0.05,0.1$.

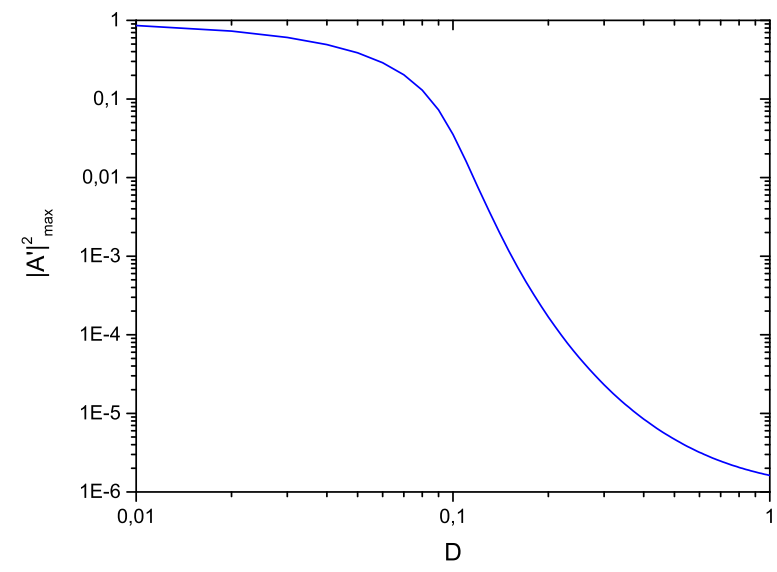

FIG. 4. $\left|A^{\prime}\right|_{\max }^{2}$ as a function of $D$, calculated from Eqs. (18) to (21) and $\delta^{\prime}=0$.

spontaneous emission quenches the coherent FEL lasing when $D>0.2$

We complete the analysis by presenting the result of the numerical integration of the full Eqs. (8) and (9), valid in the

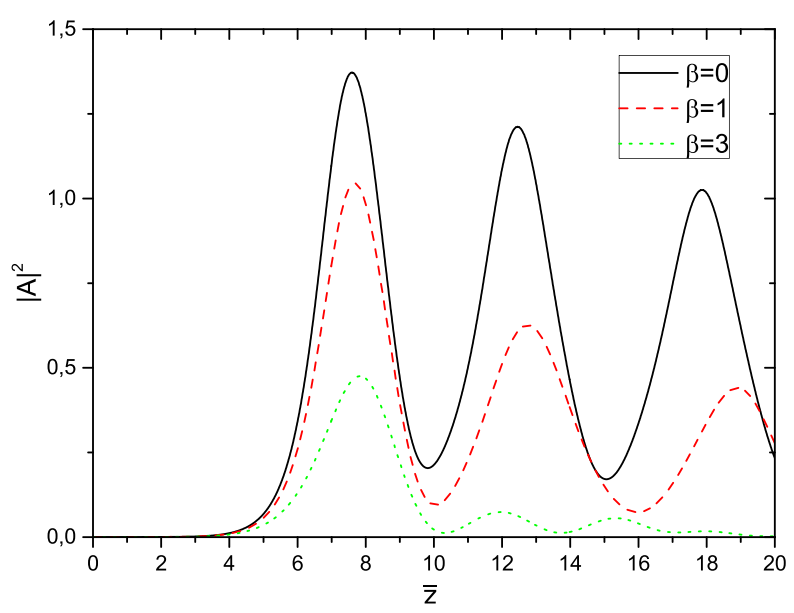

FIG. 5. $|A|^{2}$ vs. $\bar{z}$ for $\bar{\rho}=5, \delta=0$, and $\beta=0,1,3$. 


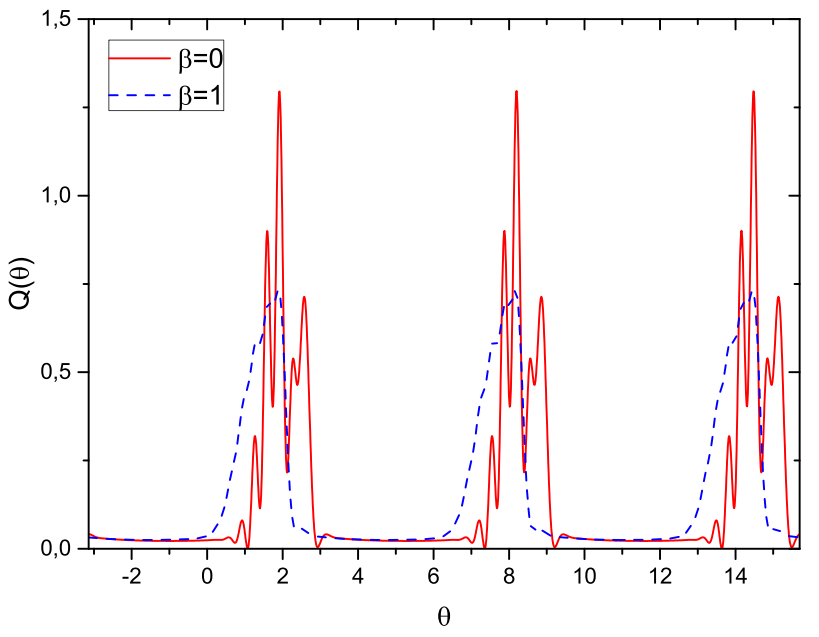

FIG. 6. $Q(\theta)$ vs. $\theta$ at $\bar{z}=7$, for $\bar{\rho}=5, \delta=0$, and $\beta=0,1$.
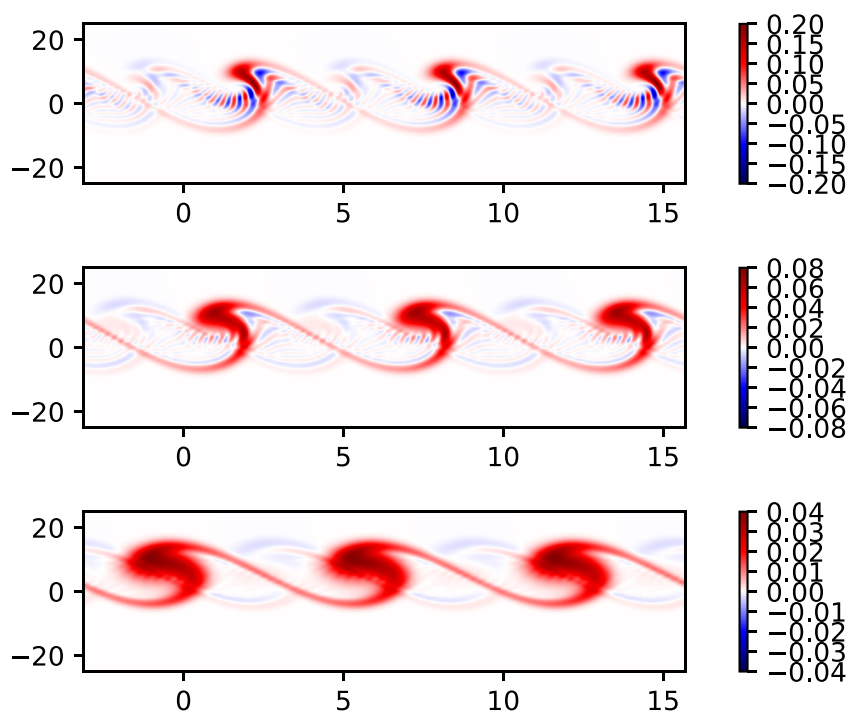

FIG. 7. Phase-space quasi-distribution $W_{m}(\theta)$ vs. $\theta$ and $m$ for $\beta=0$ (up, at $\bar{z}=6.96$ ), $\beta=1$ (middle, at $\bar{z}=7.13$ ), and $\beta=3$ (bottom, at $\bar{z}=7.57$ ). The other parameters are $\bar{\rho}=5$ and $\delta=0$.

general case of arbitrary $\bar{\rho}$. Figure 5 shows the scaled intensity $|A|^{2}$ vs. $\bar{z}$ for $\bar{\rho}=5, \delta=0$ and $\beta=0$ (black full line), $\beta=1$ (red dashed line) and $\beta=3$ (green dotted line). This value of $\bar{\rho}$ corresponds to a quasi-classical regime, with several photons emitted per electron. We observe that the emission is severely inhibited already for $\beta=3$. Figure 6 shows the distribution $Q(\theta)$ vs. $\theta$ at $\bar{z}=7$ for the same parameters as in Fig. 5, for $\beta=0$ (red continuous line) and for $\beta=1$ (blue dashed line). We observe that the energy spread induced by the spontaneous emission smears the electron spatial distribution. This behaviour is more evident in Fig. 7 showing the electron phase-space distribution described by the discrete Wigner function $W_{m}(\theta)$, at the positions where the maximum bunching occurs for $\beta=0,1$, and 3 . The phase-space distribution of the micro-bunches tends to be less filamented and jagged. Notice the zone in the phasespace where the quasi-distribution is negative, an indicator of a non-classical behavior.

\section{CONCLUSIONS}

We have presented a discrete Wigner model for the quantum FEL, including spontaneous emission. This model is more rigorous and formally correct than the continuous Wigner model presented in Ref. 11, since it describes the momentum as a discrete variable, as it should be assuming spatial periodic boundary conditions. However, the results are in good agreement with those of the continuous model, as it can be observed comparing Fig. 4 with Fig. 6 of Ref. 11. We have shown that, in the quantum regime, the equations reduce to these for two-momentum states coupled to the coherent radiation field. Spontaneous emission is therefore interpreted as responsible for the loss of coherence (i.e., bunching) and the transfer of electrons in and out of the two momentum states via rate equation terms.

\section{ACKNOWLEDGMENTS}

This article is dedicated to the memory of Rodolfo Bonifacio, who introduced us to the concept of the quantum free-electron laser. One of the authors, H. Fares, would like to acknowledge support from the Academy of Scientific Research and Technology (ASRT) in Egypt and INFN in Italy (ASRT-INFN joint project).

${ }^{1}$ C. Bostedt, J. D. Bozek, P. H. Bucksbaum, R. N. Coffe1, J. B. Hastings, Z. Huang, R. W. Le1, S. Schorb, J. N. Corlett, P. Denes et al., J. Phys. B 46, 164003 (2013).

${ }^{2}$ T. Ishikawa, H. Aoyagi, T. Asaka, Y. Asano, N. Azumi, T. Bizen, H. Ego, K. Fukami, T. Fukui, Y. Furukawa et al., Nat. Photonics 6, 540 (2012).

${ }^{3}$ V. Petrillo, L. Serafini, and P. Tomassini, Phys. Rev. ST Accel. Beams 11, 070703 (2008)

${ }^{4}$ J. Bahrdt and Y. Ivanyushenkov, J. Phys.: Conf. Ser. 425, 032001 (2013).

${ }^{5}$ R. Bonifacio, N. Piovella, and G. R. M. Robb, Nucl. Instrum. Methods Phys. Res., Sect. A 543, 645 (2005).

${ }^{6}$ R. Bonifacio, N. Piovella, G. R. M. Robb, and A. Schiavi, Phys. Rev. Spec. Top. -Accel. Beams 9, 090701 (2006).

${ }^{7}$ R. Bonifacio, C. Pellegrini, and L. M. Narducci, Opt. Commun. 50, 373 (1984).

${ }^{8}$ S. Benson and J. M. J. Madey, Nucl. Instrum. Methods Phys. Res., Sect. A 237, 55 (1985)

${ }^{9}$ E. J. Saldin, E. A. Schneidmiller, and M. V. Yurkov, Nucl. Instrum. Methods Phys. Res., Sect. A 381, 545 (1996).

${ }^{10}$ G. R. M. Robb and R. Bonifacio, EPL 94, 34002 (2011); G. Geloni, V. Kocharyan, and E. Saldin, ibid. 98, 44001 (2012); A. Potylitsyn and A. Kol'chuzhkin, ibid. 100, 24006 (2012); G. R. M. Robb and R. Bonifacio, ibid. 98,44002 (2012).

${ }^{11}$ G. R. M. Robb and R. Bonifacio, Phys. Plasmas 19, 073101 (2012).

${ }^{12}$ G. R. M. Robb and R. Bonifacio, Phys. Plasmas 20, 033106 (2013); V. Petrillo, A. R. Rossi, and L. Serafini, ibid. 20, 124701 (2013); G. R. M. Robb and R. Bonifacio, ibid. 20, 124702 (2013).

${ }^{13}$ G. A. Krafft and G. Priebe, Rev. Accel. Sci. Tech. 3, 147 (2010).

${ }^{14}$ C. Sun and Y. K. Wu, Phys. Rev. Spec. Top. Accel. Beams 14, 044701 (2011).

${ }^{15}$ C. Curatolo, I. Drebot, V. Petrillo, and L. Serafini, Phys. Rev. Spec. Top. Accel. Beams 20, 080701 (2017).

${ }^{16}$ J. P. Bizarro, Phys. Rev. A 49, 3255 (1994).

${ }^{17}$ N. Piovella, M. M. Cola, L. Volpe, R. Gaiba, A. Schiavi, and R. Bonifacio, Opt. Commun. 274, 347 (2007).

${ }^{18}$ J. D. Jackson, Classical Electrodynamics, 3rd ed. (John Wiley \& Sons, 1998), p. 683.

${ }^{19}$ R. Bonifacio and G. Preparata, Phys. Rev. A 2, 336 (1970).

${ }^{20}$ M. O. Scully and M. S. Zubairy, Quantum Optics (Cambridge University Press, 1997), p. 164. 